\title{
Effects of Toroidal Field Ripples on the Bootstrap Current in Tokamaks
}

\author{
Yuji NAKAMURA \\ Graduate School of Energy Science, Kyoto University, Uji 611-0011, Japan
}

(Received 14 December 2005 / Accepted 26 January 2006)

\begin{abstract}
The effects of toroidal field ripples on bootstrap current in the low collisionality regime in tokamak plasmas are discussed by using an asymptotic expression of the bootstrap current. When the toroidal field ripple is $1-3 \%$, an $8-15 \%$ reduction of the bootstrap current is predicted in a tokamak plasma having $q=1.8$ and $\varepsilon_{\mathrm{t}}=0.3$. If we fix the ripple ratio, dependence on the number of toroidal field coils is negligible when the number is more than 15. These results suggest that the effect of the toroidal field ripple on the bootstrap current is particularly important when the pressure gradient is steep near the plasma edge.
\end{abstract}

(c) 2006 The Japan Society of Plasma Science and Nuclear Fusion Research

Keywords: bootstrap current, ripple, tokamak, neoclassical theory, low collisionality regime

DOI: $10.1585 /$ pfr. 1.010

In most previous analyses of bootstrap currents based on the neoclassical transport theory, a tokamak plasma is considered to be axisymmetric. Since an actual tokamak is nonaxisymmetric due to toroidal field (TF) ripples produced by the discreteness of TF coils, it is important to estimate the effect of the TF ripples on the bootstrap current in a tokamak plasma. It is natural to speculate that TF ripples reduce both the bootstrap currents and the neoclassical parallel flows which are driven by the parallel viscosity because the TF ripples create symmetry-breaking components of the magnetic field strength.

An asymptotic approach to the bootstrap current in nonaxisymmetric toroidal plasmas was described by Shaing and Callen [1], and its analytic expression in Boozer coordinates is given for the low collisionality regime by Shaing et al. [2]. Watanabe et al. [3,4] used this expression to calculate the bootstrap current for LHD [5] plasmas. In the present study, we use the BSC code [4] to estimate the bootstrap currents in rippled tokamaks.

As is shown in Refs. [1-4], the bootstrap current's dependence on the magnetic configuration can be expressed in terms of the geometric factor $[2,3] \tilde{G}_{\mathrm{b}}$. The geometric factor can be calculated from the total poloidal current outside a flux surface $G$, the total toroidal current inside a flux surface $I$, the safety factor $q$, and the distribution of the magnetic field strength $B(\theta, \zeta)$ on a flux surface, and can be expressed in Boozer coordinates as [2,3]

$$
\tilde{G}_{\mathrm{b}}=\tilde{G}_{\mathrm{b}}^{\mathrm{H}_{1}}+\tilde{G}_{\mathrm{b}}^{\mathrm{H}_{2}}+\tilde{G}_{\mathrm{b}}^{\mathrm{W}},
$$

where

$$
\begin{aligned}
& \tilde{G}_{\mathrm{b}}^{\mathrm{H}_{1}}=\left\langle H_{1}\right\rangle=(q G-I) / 2, \\
& \tilde{G}_{\mathrm{b}}^{\mathrm{H}_{2}}=H_{2}(q G+I) / 2,
\end{aligned}
$$

author'se-mail: nakamura@energy.kyoto-u.ac.jp

$$
\begin{aligned}
& \tilde{G}_{\mathrm{b}}^{\mathrm{W}}=-\frac{3}{4} \frac{q(q G+I)}{f_{\mathrm{t}}} \int_{0}^{1} \frac{\lambda W(\lambda) \mathrm{d} \lambda}{\left\langle\sqrt{1-\lambda B / B_{\max }}\right.}, \\
& H_{2}=\frac{\left\langle(\partial B / \partial \theta)^{2}\right\rangle-q^{2}\left\langle(\partial B / \partial \zeta)^{2}\right\rangle}{\left\langle(\partial B / \partial \theta+q \partial B / \partial \zeta)^{2}\right\rangle},
\end{aligned}
$$

$\theta(\zeta)$ is the poloidal (toroidal) angle in Boozer coordinates, and $\langle\cdot\rangle$ indicates the flux surface averaged quantity. The fraction of trapped particles $f_{\mathrm{t}}$ and $W(\lambda)$, whose explicit expressions are given in Ref. [3], are calculated from $B(\theta, \zeta)$. The term $\tilde{G}_{\mathrm{b}}^{\mathrm{W}}$ vanishes if the magnetic filed strength's distribution on a flux surface has any symmetry [6], such as axisymmetry or helical symmetry. Note that $\tilde{G}_{\mathrm{b}}^{\mathrm{H}_{2}}$ also contains asymmetric contribution through $\mathrm{H}_{2}$, which is closely related to the direction of the grad-B drift, and it plays a dominant role in determininig the direction of the bootstrap current. If the configuration is axisymmetric, $B=B(\theta), H_{2}$ becomes unity and $\tilde{G}_{\mathrm{b}}^{\text {tok }}=q G$ can be obtained. This shows that all axisymmetric tokamaks have the same geometric factor whether they have a circular or D-shaped cross section, for example.

Now let us consider a rippled tokamak whose magnetic field strength is written by $B(\theta, \zeta)=B_{0}\left(1-\varepsilon_{\mathrm{t}} \cos \theta+\right.$ $\delta \cos (N \zeta))$. In this case, $H_{2}$ becomes $\left(\varepsilon_{\mathrm{t}}^{2}-N^{2} q^{2} \delta^{2}\right) /\left(\varepsilon_{\mathrm{t}}^{2}+\right.$ $\left.N^{2} q^{2} \delta^{2}\right)$, and $H_{2} \sim 1$ for $\varepsilon_{\mathrm{t}} /(N q \delta) \gg 1$. Therefore, it is remarked in Ref. [6] that a rippled tokamak with $\varepsilon_{\mathrm{t}} /(N q \delta) \gg$ 1 has parallel viscosity-driven fluxes and bootstrap currents similar to those of the axisymmetric tokamak if we neglect the effect of the boundary layer, $\tilde{G}_{\mathrm{b}}^{\mathrm{W}}$. When we consider a typical rippled tokamak with $\varepsilon_{\mathrm{t}} \sim 0.3, q \sim 2, N \sim 18$, and $\delta \sim 0.01$, however, we can get $\varepsilon_{\mathrm{t}} /(N q \delta) \sim 0.83$ and $H_{2} \sim-0.2$. This produces a significant reduction of the bootstrap current; $\tilde{G}_{\mathrm{b}} / \tilde{G}_{\mathrm{b}}^{\text {tok }} \sim\left(\tilde{G}_{\mathrm{b}}^{\mathrm{H}_{1}}+\tilde{G}_{\mathrm{b}}^{\mathrm{H}_{2}}\right) / \tilde{G}_{\mathrm{b}}^{\text {tok }} \sim 0.3$ (hereafter, we neglect $I$ in equations because $I / q G \ll 1$ is satisfied in most tokamak plasmas). This means that small 

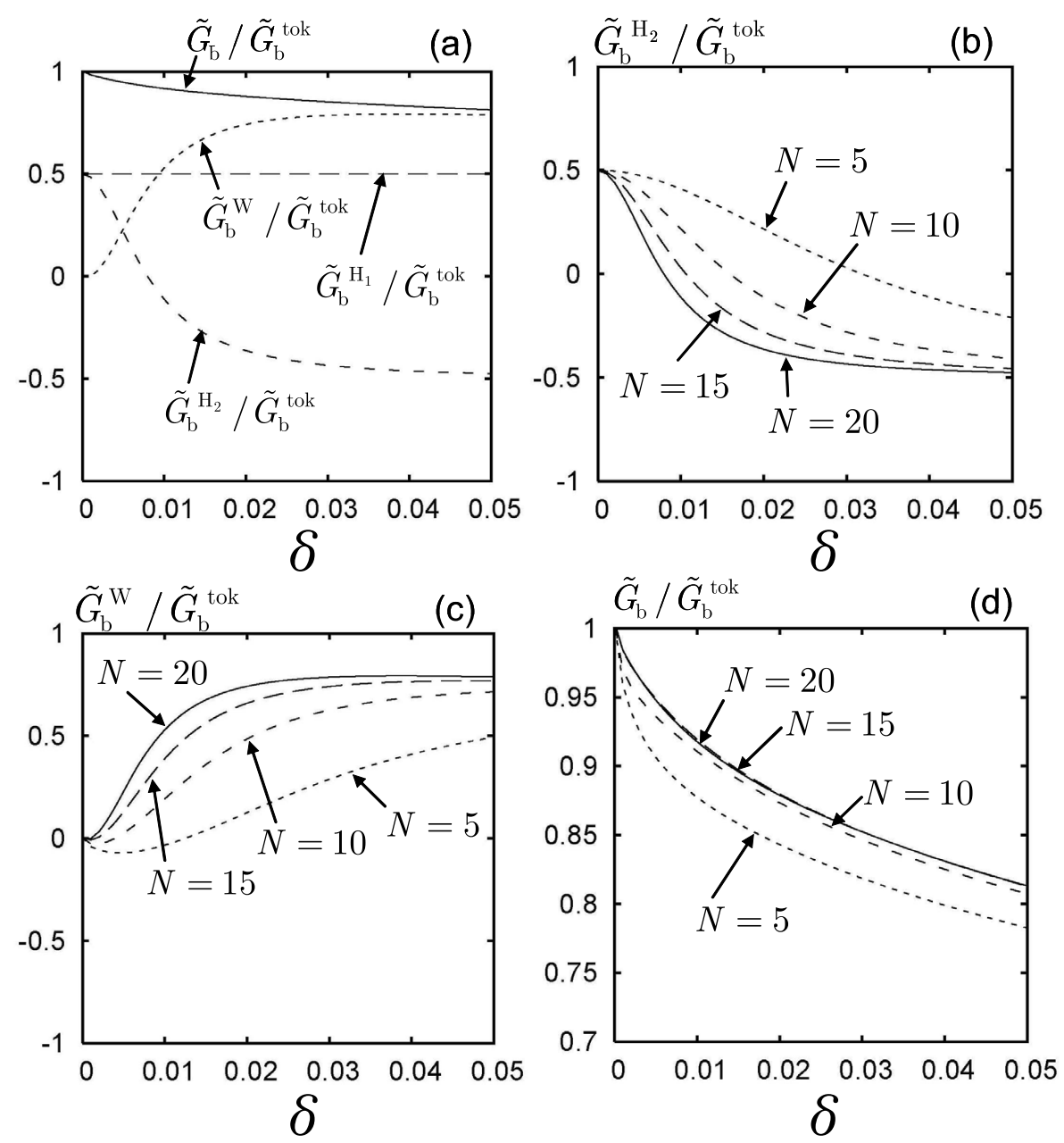

Fig. 1 TF ripple dependence of the normalized geometric factor for rippled tokamaks with $\varepsilon_{\mathrm{t}}=0.3, q=1.8$ : (a) components of $\tilde{G}_{\mathrm{b}} / \tilde{G}_{\mathrm{b}}^{\text {tok }}$ for $N=20$, (b) $\tilde{G}_{\mathrm{b}}^{\mathrm{H}_{2}} / \tilde{G}_{\mathrm{b}}^{\mathrm{tok}}$ component for $N=5,10,15,20$, (c) $\tilde{G}_{\mathrm{b}}^{\mathrm{W}} / \tilde{G}_{\mathrm{b}}^{\text {tok }}$ component for $N=5,10,15,20$, (d) geometric factor $\tilde{G}_{\mathrm{b}} / \tilde{G}_{\mathrm{b}}^{\text {tok }}$ for $N=5,10,15,20$.

symmetry-breaking components such as TF ripples might reduce the bootstrap currents to a notable degree.

Since the bootstrap current is very sensitive to the physics of the trapped-untrapped boundary, we should take great care in estimating of the bootstrap current. In the present study, we calculate the geometric factor of the rippled tokamak numerically by taking account of the boundary layer effect, $\tilde{G}_{\mathrm{b}}^{\mathrm{W}}$, in order to qualitatively estimate the effect of TF ripples.

Figure 1 (a) shows the dependence of the geometric factor on the TF ripple $\delta$ in rippled tokamaks with $\varepsilon_{\mathrm{t}}=0.3$, $q=1.8$, and $N=20$. As noted above, dependence of the $\tilde{G}_{\mathrm{b}}^{\mathrm{H}_{2}}$ on the TF ripple is quite strong, and simple estimation of the geometric factor, $\left(\tilde{G}_{\mathrm{b}}^{\mathrm{H}_{1}}+\tilde{G}_{\mathrm{b}}^{\mathrm{H}_{2}}\right) / \tilde{G}_{\mathrm{b}}^{\text {tok }}$, is almost zero for $\delta \geqslant 0.03$ as in the poloidally symmetric case. However, it can be seen from Fig. 1 (a) that $\tilde{G}_{\mathrm{b}}^{\mathrm{W}}$ compensates for the change of $\tilde{G}_{\mathrm{b}}^{\mathrm{H}_{2}}$ due to asymmetry, and it suppresses the reduction of the bootstrap current. This result shows that TF ripples of $\delta=1 \%$ cause an $8 \%$ reduction of the bootstrap current and that those of $\delta=3 \%$ cause a $15 \%$ reduction. Though these reductions are not so drastic than those by the previous estimation, they are still at a level that cannot be ignored. Figure 1 (b) shows the TF ripple dependence of $\tilde{G}_{\mathrm{b}}^{\mathrm{H}_{2}} / \tilde{G}_{\mathrm{b}}^{\text {tok }}$ for rippled tokamaks with $N=5$, 10,15 , and 20. As we can see from the definition, $\tilde{G}_{\mathrm{b}}^{\mathrm{H}_{2}}$ depends on the number of TF coils $N$. However, $\tilde{G}_{\mathrm{b}}^{\mathrm{W}}$ also changes with $N$ so as to compensate for the change of $\tilde{G}_{\mathrm{b}}^{\mathrm{H}_{2}}$ (Fig. 1 (c)). As a result, the $N$ dependence of the geometric factor becomes moderate (Fig. 1 (d)), and the $N$ dependence can be neglected for usual tokamak plasmas with $N \geqslant 15$. It should be noted that the $N$ dependence can appear through $\delta$, in practice, because lower $N$ gives larger $\delta$ in a realistic equilibrium.

In this study, the importance of TF ripples on the bootstrap current estimation is demonstrated in a tokamak. These results suggest that the effect of the TF ripple on the bootstrap current is particularly important when the pressure gradient is steep near the plasma edge. If we lower the toroidicity $\varepsilon_{\mathrm{t}}$, reduction of the bootstrap current due to TF ripples is more pronounced. We also found that the ef- 
fect of the safety factor on bootstrap current reduction due to TF ripples is weak. Detailed parameter dependence and the impact of the bootstrap current reduction on the MHD equilibrium will be reported in a separate article.

This work is supported by the 21 st Century COE program "Establishment of COE on Sustainable Energy System" from MEXT, Japan.

[1] K.C. Shaing and J.D. Callen, Phys. Fluids 26, 3315 (1983).

[2] K.C. Shaing, B.A. Carreras, N. Dominguez, V.E. Lynch and J.S. Tolliver, Phys. Fluids B 1, 1663 (1989).

[3] K. Watanabe, N. Nakajima, M. Okamoto, Y. Nakamura and M. Wakatani, Nucl. Fusion 32, 1499 (1992).

[4] K.Y. Watanabe, N. Nakajima, M. Okamoto, K. Yamazaki, Y. Nakamura and M. Wakatani, Nucl. Fusion 35, 335 (1995).

[5] A. Iiyoshi, M. Fujiwara, O. Motojima, N. Ohyabu and K. Yamazaki, Fusion Technol. 17, 169 (1990).

[6] K.C. Shaing, S.P. Hirshman and J.S. Tolliver, Phys. Fluids 29, 2548 (1986). 